\title{
La restructuration du commerce de détail en Saxe - où en sont les centres des grandes villes?
}

Restructuring of the retail trade in Saxonia - what is the fate of city centres?

Umstrukturierung des Einzelhandels in Sachsen - wo bleiben die Innenstädte?

\section{Maike Hoppmann et Helga Schmidt}

\section{OpenEdition}

\section{Journals}

Édition électronique

URL : http://journals.openedition.org/rge/3793

DOI : $10.4000 /$ rge.3793

ISSN : 2108-6478

Éditeur

Association des géographes de l'Est

Édition imprimée

Date de publication : 1 juin 2001

ISSN : 0035-3213

Référence électronique

Maike Hoppmann et Helga Schmidt, "La restructuration du commerce de détail en Saxe - où en sont les centres des grandes villes ? », Revue Géographique de l'Est [En ligne], vol. 41 / 3 | 2001, mis en ligne le 09 juillet 2013, consulté le 08 septembre 2020. URL : http://journals.openedition.org/rge/3793

DOI : https://doi.org/10.4000/rge.3793

Ce document a été généré automatiquement le 8 septembre 2020.

Tous droits réservés 


\section{La restructuration du commerce de détail en Saxe - où en sont les centres des grandes villes?}

Restructuring of the retail trade in Saxonia - what is the fate of city centres? Umstrukturierung des Einzelhandels in Sachsen - wo bleiben die Innenstädte?

Maike Hoppmann et Helga Schmidt

\section{Introduction}

Depuis 1990 le commerce de détail dans les nouveaux Länder a été complètement restructuré. Cette restructuration (déterminée par un engagement fort des entreprises commerciales ouest-allemandes) a été marquée par une énorme croissance des surfaces de vente sur des localisations périurbaines, tandis que les villes-centres enregistraient une forte perte de fonction. En Saxe également les villes-centres ont été perdantes (Jürgens 1998). Depuis 1996 on peut observer dans les grandes viles saxonnes ce qui apparaît par bien des aspects comme une renaissance des centres-villes liée à un réaménagement pour le commerce et la promenade. Plus de 10 ans après la réunification de l'Allemagne, cette contribution s'intéresse à la structure de localisation du commerce de détail dans les grandes villes, Dresde, Leipzig et Chemnitz.

\section{Les conditions de la transformation : une diversité socialiste dans le commerce de détail ?}

2 Le réseau commercial de détail de l'ancienne RDA était soumis à la planification, les décisions relatives à l'implantation étaient prises par des commissions d'état et transmises aux entreprises commerciales. Les entreprises étaient des Konzerns commerciaux d'état et des coopératives de consommation contrôlées par l'Etat (Dütz 1998). Les autorités communales n'avaient alors qu'une fonction d'exécution et 
mettaient en œuvre les directives prises au niveau central. Il ne leur était pas possible d'intervenir sur la planification du commerce de détail. L'objectif premier de l'approvisionnement commercial était alors d'assurer les besoins fondamentaux de la population. Même les entreprises commerciales possédaient peu de liberté de décision : l'assortiment de marchandises et les prix étaient fixés au niveau central, les surfaces de vente étaient attribuées par la planification centrale. En conséquence, la diversité des formes d'entreprises du commerce de détail était-elle assez faible (Pütz 1998).

3 Les nombreux petits magasins de moins de $100 \mathrm{~m}^{2}$ de surface de vente étaient typiques du commerce de détail dans l'ancienne RDA. En 1988, la dimension moyenne des magasins était d'environ $68 \mathrm{~m}^{2}$, à la même époque elle atteignait en moyenne $200 \mathrm{~m}^{2}$ dans les anciens Länder. En conséquence, la taille moyenne des magasins n'était que de $0,31 \mathrm{~m}^{2}$ par habitant alors qu'en comparaison, dans les anciens Länder, elle était de 1,07 $\mathrm{m}^{2}$ de surface de vente par habitant (Kulke 1997). Le réseau de commerce de détail était organisé en trois niveaux concentriques suivant lesquels se répartissaient les marchandises et les services en fonction de la fréquence de la demande :

1. demande permanente (journalière)

2. demande fréquente (périodique)

3. demande rare (épisodique)

4 Cette division visait à assurer une répartition équitable des marchandises, surtout en ce qui concerne l'approvisionnement de base pour les besoins quotidiens et la réalisation de conditions de vie comparables dans les régions urbaines et dans l'espace rural. Les marchandises demandées quotidiennement étaient mises à disposition partout, les marchandises demandées rarement étaient proposées de façon concentrée et centralisée dans les centres-villes. Dans les villes prévalait le principe de proximité (la ville des cheminements réduits) suivant lequel on devait accéder au lieu de vente en 8 à 15 minutes à pied.

5 Le système commercial de la RDA, tout comme celui de l'Allemagne de l'Ouest, a subi un changement de structure qui, toutefois, n'a pas été dirigé par les entreprises, mais a été initié par l'état. Le système commercial a dû ainsi s'adapter aux demandes changeantes de consommation de la population. Après que l'état ait fait reculer l'importance du secteur commercial privé, au début des années 50 , le commerce a été libéralisé à partir du début des années 70. Par souci de rationalisation, de grandes surfaces de vente comparables aux supermarchés des anciens Länder sont développées par l'état à partir du début des années 60 . Avec ces surfaces de vente appelées « Kaufhallen » on a essayé d'agrandir la taille des entreprises et de diminuer le nombre de magasins.

6 À la fin des années 80 , certaines formes de commerce privées étaient soutenues par l'état, afin d'assurer l'approvisionnement de la population (Jürgens 1998). Le commerce privé était cependant rarement soutenu ou encouragé dans les centres-villes, mais plutôt principalement dans des implantations périphériques peu attractives, comme par exemple dans les quartiers du Gründerzeit des grandes villes saxonnes (Pütz 1997). Dans les rues commerçantes des centres-villes se sont développés des grands magasins constituant des pôles d'attraction pour les clients. Dans les nouveaux quartiers d'habitat, on a construit des centres commerciaux que l'on peut comparer aux centres commerciaux de quartier d'Allemagne de l'Ouest. Ces infrastructures n'avaient de toute façon qu'une importance locale (Pütz 1997). 
7 Au moment de la réunification, la structure du réseau de commerce de détail se caractérisait de la façon suivante: des petites surfaces de vente d'état, distribuées régulièrement dans la ville à des intervalles d'un quart d'heure à pied en fonction du principe de proximité. Les magasins étaient dans l'ensemble très petits, atteignant au plus $100 \mathrm{~m}^{2}$, mis à part les "Kaufhallen " et les magasins spécialisés dans les rues commerçantes des centres-villes. Une caractéristique générale était le fort délabrement du bâti.

8 Les entreprises commerciales ouest-allemandes qui, dès 1989, ont fait massivement irruption dans le tissu commercial des nouveaux Länder, se sont retrouvées devant le problème suivant : si il y avait suffisamment de magasins dans les villes, ceux-ci ne répondaient pas aux exigences et surtout ne correspondaient pas aux dimensions des équipements commerciaux modernes.

\section{L'évolution de la localisation du commerce de détail en saxe depuis la réunification et ses conséquences sur les centres-villes}

9 Les premières années après la réunification ont été marquées par un renforcement de l'implantation des entreprises commerciales ouest-allemandes sur des sites périphériques non intégrés. Comment en est-on arrivé là ? Le total des surfaces de vente disponibles dans les nouveaux Länder s'élève à 18 millions de $\mathrm{m}^{2}$, les deux tiers se trouvant en dehors des centres-villes (fig. 1 et tableau 1). Il s'est produit une véritable course vers l'extérieur au détriment des centres-villes et en particulier des centres d'approvisionnement de proximité (Stadtplanungsamt Leipzig et GMA 1999). En raison de la politique de développement urbain de l'ancienne RDA, les villes saxonnes, et en particulier les centres-villes étaient, comme on l'a vu plus haut, caractérisés par un bâti dégradé avec de petits magasins. Le caractère délabré des centres, par exemple du centre de Leipzig, peut avoir exercé un certain charme sur le visiteur, mais c'était tout à fait inadapté à l'établissement de commerces modernes. Aussi, dans les premières années, il n'a pas été question d'installer de grandes surfaces commerciales dans les centres-villes et le déclin de ceux-ci s'est reflété dans la diminution du flot des passants fréquentant les rues commerçantes. Les implantations recherchées se sont limitées aux lieux situés en position non intégrée, possédant néanmoins les avantages suivants par rapport aux villes (Jürgens 1998, Pütz 1998, Schmidt \& Fliegner 1997) :

10 - Ils étaient immédiatement disponibles et les prix des terrains étaient nettement plus bas que dans les villes-centres.

11 - La construction de nouveaux bâtiments revenait moins cher que la réhabilitation de l'ancien bâti qui devait être réalisée en tenant compte des règles de protection du patrimoine historique des centres-villes.

12 - Les maires des petites communes périphériques saluèrent les désirs d'installation des entreprises en espérant des emplois, la garantie d'une amélioration des infrastructures et un afflux de ressources fiscales. Surtout, après des années d'impuissance des communes, ils avaient enfin la chance de participer au remodelage de leur région. 
Figure 1 : Les grandes surfaces commerciales dans les grandes villes saxonnes et leurs alentours en 1998

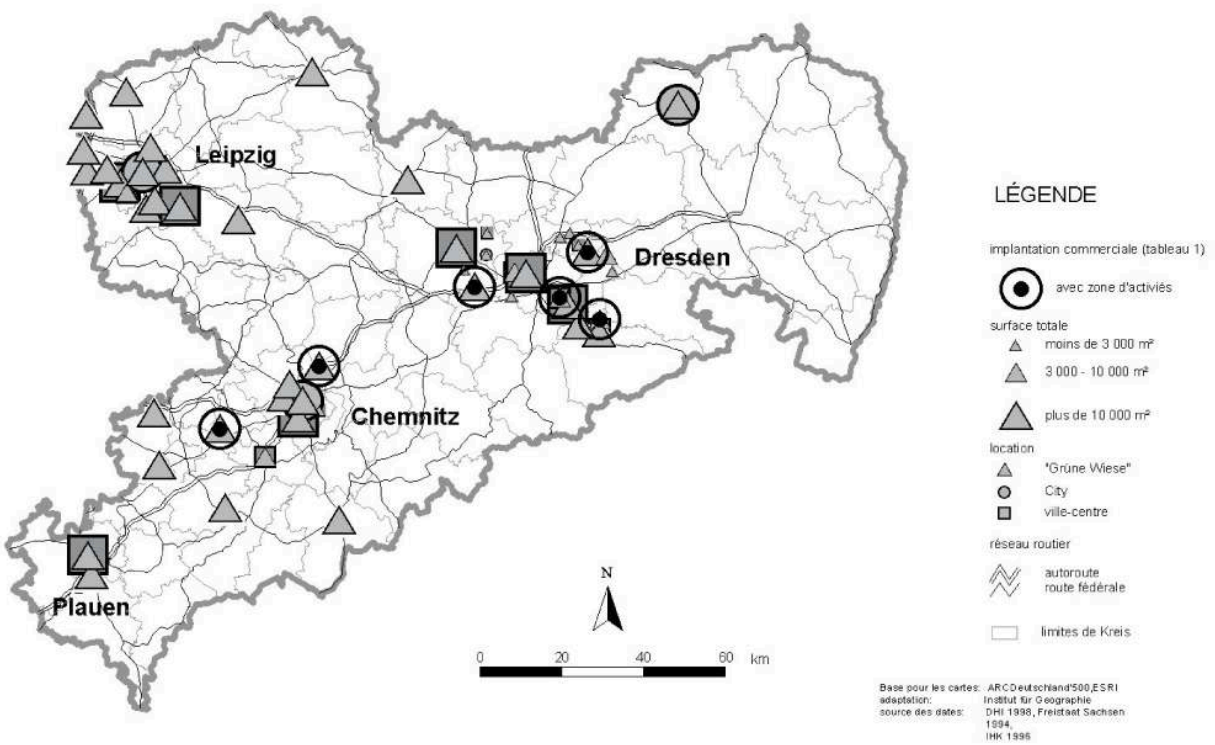

Tableau 1 : Construction de centres commerciaux en Saxe depuis 1990

\begin{tabular}{|c|c|c|c|c|c|}
\hline $\begin{array}{l}\text { Nom du centre } \\
\text { commercial }\end{array}$ & Lieu & Ouverture & Position & Surface totale & Surface de vente \\
\hline Elbe Park & Dresden & 1995 & urbaine & $70000 \mathrm{~m}^{2}$ & $62820 \mathrm{~m}^{2}$ \\
\hline Görbitz-Center & Dresden-Görbitz & 1994 & urbaine & $15000 \mathrm{~m}^{2}$ & $11770 \mathrm{~m}^{2}$ \\
\hline Sachsen-Forum & Dresden-Görbitz & 1996 & urbaine & $13500 \mathrm{~m}^{2}$ & $10880 \mathrm{~m}^{2}$ \\
\hline Kaufpark & Dresden & 1996 & urbaine & $60000 \mathrm{~m}^{2}$ & $45000 \mathrm{~m}^{2}$ \\
\hline Seidnitz-Center & Dresden & 1994 & urbaine & $38720 \mathrm{~m}^{2}$ & $20578 \mathrm{~m}^{2}$ \\
\hline Riesa-Park & Riesa & 1993 & Grüne Wiese & $48100 \mathrm{~m}^{2}$ & $45734 \mathrm{~m}^{2}$ \\
\hline Elbe-Center & Meißen & 1994 & urbaine & $13500 \mathrm{~m}^{2}$ & $11030 \mathrm{~m}^{2}$ \\
\hline Lausitz-Center & Hoyerswerda & 1995 & centre-ville & $13000 \mathrm{~m}^{2}$ & $11000 \mathrm{~m}^{2}$ \\
\hline Promenaden $\mathrm{Hbf}$ & Leipzig & 1997 & Centre-ville & $36000 \mathrm{~m}^{2}$ & $27000 \mathrm{~m}^{2}$ \\
\hline Allee-Center & Leipzig & 1996 & urbaine & $29000 \mathrm{~m}^{2}$ & $24000 \mathrm{~m}^{2}$ \\
\hline Paunsdorf-Center & Leipzig & 1994 & Grüne Wiese & $110000 \mathrm{~m}^{2}$ & $104176 \mathrm{~m}^{2}$ \\
\hline Löwen-Center & Burghausen & 1993 & Grüne Wiese & $47075 \mathrm{~m}^{2}$ & $41672 \mathrm{~m}^{2}$ \\
\hline Sachsenpark & Seehausen & 1992 & Grüne Wiese & $36000 \mathrm{~m}^{2}$ & $30400 \mathrm{~m}^{2}$ \\
\hline Pösna-Park & Großpösna & 1993 & urbaine & $56530 \mathrm{~m}^{2}$ & $51825 \mathrm{~m}^{2}$ \\
\hline PEP Delitzsch & Delitzsch & 1994 & Grüne Wiese & $19000 \mathrm{~m}^{2}$ & $16935 \mathrm{~m}^{2}$ \\
\hline PEP Grimma & Grimma & 1994 & Grüne Wiese & $17000 \mathrm{~m}^{2}$ & $14600 \mathrm{~m}^{2}$ \\
\hline PEP Torgau & Torgau & 1994 & Grüne Wiese & $27800 \mathrm{~m}^{2}$ & $20699 \mathrm{~m}^{2}$ \\
\hline Pleissen-Center & Steinpleiß & 1992 & Grüne Wiese & $35600 \mathrm{~m}^{2}$ & $21539 \mathrm{~m}^{2}$ \\
\hline $\begin{array}{l}\text { LCC-Langenbahn- } \\
\text { Center Crimmitschau }\end{array}$ & Crimmitschau & 1995 & Grüne Wiese & $23500 \mathrm{~m}^{2}$ & $22000 \mathrm{~m}^{2}$ \\
\hline Plauen Park & Plauen & 1994 & Grüne Wiese & $31810 \mathrm{~m}^{2}$ & $17630 \mathrm{~m}^{2}$ \\
\hline Elster Park & Plauen & 1995 & urbaine & $25000 \mathrm{~m}^{2}$ & $23816 \mathrm{~m}^{2}$ \\
\hline Neefe-Park & Chemnitz & 1994 & Grüne Wiese & $30300 \mathrm{~m}^{2}$ & $22772 \mathrm{~m}^{2}$ \\
\hline Alt-Chemnitz-Center & Chemnitz & 1993 & urbaine & $60000 \mathrm{~m}^{2}$ & $46061 \mathrm{~m}^{2}$ \\
\hline Sachsen-Allee & Chemnitz & 1997 & centre-ville & $38000 \mathrm{~m}^{2}$ & $32000 \mathrm{~m}^{2}$ \\
\hline Chemnitz-Center & Röhrsdorf & 1992 & Grüne Wiese & $82540 \mathrm{~m}^{2}$ & $69485 \mathrm{~m}^{2}$ \\
\hline Erzgebirgs-Center & Annaberg-Buchholz & 1995 & Grüne Wiese & $45000 \mathrm{~m}^{2}$ & $36700 \mathrm{~m}^{2}$ \\
\hline
\end{tabular}

Source : EHI Fachdokumentation 1998

13 Aussi, il y a eu installation massive, et dans des proportions excessives, de nombreux grands centres commerciaux situés en dehors des villes, dans de petites communes périurbaines, en situation non intégrée, « auf der grünen Wiese ». Cela a provoqué de nombreuses fermetures. Un autre problèmes des petits commerçants de centre-ville, ce 
sont les enseignes originaires des anciens Länder qui, depuis la réunification, ont fait irruption dans les centres-villes et ont provoqué une hausse des loyers des magasins. Le taux de filialisation de Leipzig peut être considéré comme représentatif de celui des trois grandes villes. Avec environ $50 \%$ au 31/12/97 il était certes relativement faible par rapport à celui des grandes villes ouest-allemandes, mais au cours des dernières années, il a nettement augmenté aux dépens du commerce privé de détail et atteint maintenant environ $75 \%$.

14 Le tableau 1 montre qu'à partir du milieu des années 90, les centres commerciaux ont tendance à s'installer à l'intérieur des villes, alors que depuis le début des années 90 les implantations périurbaines prédominaient. D'autre part, d'une manière assez caractéristique, les centres commerciaux se sont installés surtout « auf der grünen Wiese ", c'est-à-dire en bordure de petites communes. Quels sont les impacts des centres commerciaux périurbains et des implantations " auf der grünen Wiese »? Il s'agit essentiellement d'une migration des consommateurs de la ville vers les communes rurales ayant entrainé un fort affaiblissement et même une désertification partielle des centres-villes. Les petits commerces de détail en particulier ont beaucoup souffert de la concurrence des établissements «auf der grünen Wiese ».

\section{Les stratégies de revitalisation des villes saxonnes - ville compacte contre "grüne Wiese »}

Dans les conceptions culturelles d'Europe centrale, les centres-villes ont une importance particulière; ils se sont développés comme centres de commerce et ont aujourd'hui une multitude de fonctions. L'une d'entre elles est l'image de la ville. Un centre-ville attractif est aujourd'hui associé avec des possibilités d'achat variées, une offre gastronomique, culturelle et de loisirs abondante (IHK Chemnitz 1999). Avant la réunification, les centres-villes devaient avant tout être des lieux d'habitat et des points de rencontre pour la communication politique et la formation. Pour cela, les centres-villes ont été en grande partie transformés. Des places ouvertes et modernes ont été réalisées qui ne correspondent pas à notre conception actuelle de la ville compacte.

Les trois grandes villes saxonnes ont en commun le fait que leur centre a été marqué par la rénovation urbaine socialiste (en partie, comme à Dresde et à Chemnitz, en raison des importantes destructions de la seconde guerre mondiale). Dès les années 70, la création de zones piétonnes dans les centres des grandes villes saxonnes a constitué une importante stratégie de revitalisation. Pourtant, après le changement politique de 1990, ces centres-villes représentaient des noyaux urbains peu attractifs et délabrés, si bien que les urbanistes, comme les grandes entreprises commerciales ont cherché à satisfaire les besoins de consommation de la population en implantant de grandes surfaces aux alentours ou en périphérie des villes. Les centres-villes apparaissaient trop petits pour pouvoir couvrir les besoins d'implantation et la demande en commerces. Déjà en 1993 on devait reconnaitre à Leipzig que cette stratégie consistant à délester le centre-ville de ses implantations commerciales était mauvaise. Il s'était formé des centres commerciaux autour de Leipzig, mais aussi en situation périurbaine qui retiraient à la ville son potentiel de vente. Jusqu'à la fin de 1995 on a construit environ $840000 \mathrm{~m}^{2}$ de surface de vente dans la région de Leipzig, dont $80 \%$ dans les alentours, 
ce qui a fait perdre à la ville des millions de recettes fiscales (fig. 2). À cela s'est ajouté le fait que, dans les trois centres urbains de Dresde, Chemnitz et Leipzig on ne trouve plus les produits nécessaires à la vie quotidienne et que les prix sont beaucoup plus élevés que dans les magasins « auf der grünen Wiese».

Figure 2 : Implantation des grandes surfaces commerciales dans la région urbaine de Leipzig, 1990-2000

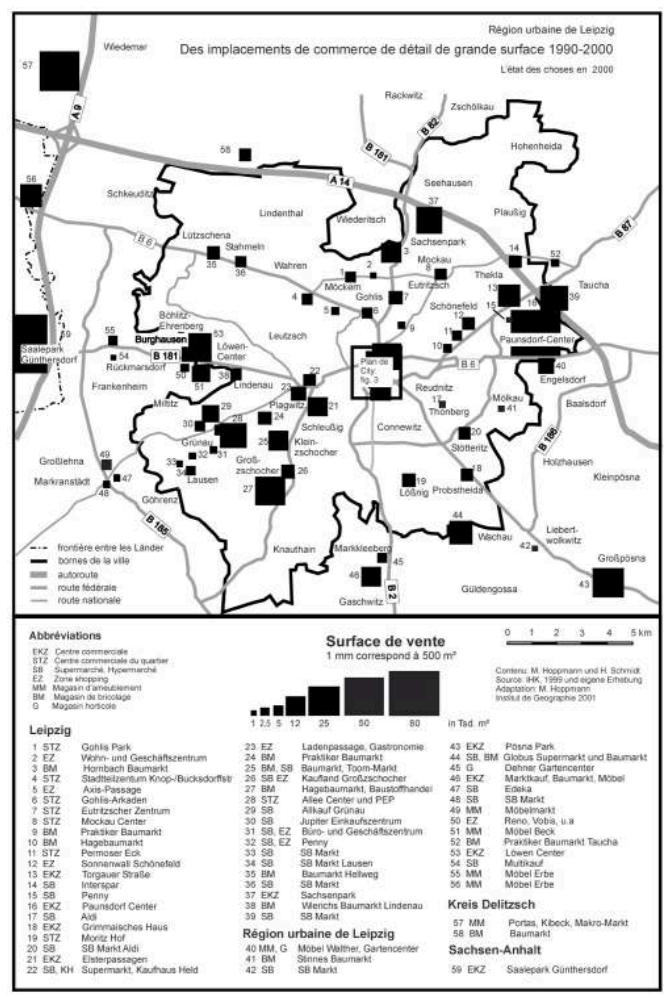

Pour combattre cette évolution, on a établi un nouveau concept qui ne prévoit plus de délestage du centre-ville, mais vise au contraire à un renforcement du centre comme lieu d'achat pour la région de Halle-Leipzig-Dessau. Quantitativement, Leipzig a un équipement commercial suffisant (surface de vente par habitant en $\mathrm{m}^{2}$ dans l'ensemble de la ville de Leipzig 1,4; dans le centre de Leipzig plus de 10. La valeur moyenne pour les centres supérieurs est de $1,2 \mathrm{~m}^{2} / \mathrm{hab}$.). Le potentiel d'approvisionnement est cependant trop faible pour un centre supérieur, puisqu'il manque la diversité correspondante du point de vue des branches et des prix. Aussi cherche-t-on à renforcer la fonction supérieure en complétant et en améliorant la qualité de la structure commerciale. Une stratégie de revitalisation des centres-villes comme celle qui est conduite à Leipzig et à Dresde (Leitbild einer polyzentralischen Einzelhandelstruktur, Dresden 1992) et depuis 1999 également à Chemnitz, prévoit la mise en place du concept des centres de quartiers dans le cadre des plans de développement urbain. Dans ce concept, la fonction directrice est dévolue au commerce de détail et à ses usages complémentaires. Les trois villes donnent la préférence à la revitalisation de leurs centres, des quartiers du Gründerzeit et des grands ensembles. Cet objectif doit être atteint par la construction ou la modernisation de centres commerciaux intégrés. À titre d'exemple, on présentera le plan de développement des centres de Leipzig (qui a été utilisé comme modèle pour la ville de Chemnitz). L'objectif premier de la politique de développement urbain est le 
renforcement de Leipzig comme centre supérieur et le développement d'une structure de centre concurrentiel. Il doit être atteint en combattant la concurrence du commerce non intégré par une politique d'implantations intégrées (Stadt Leipzig 1998). On doit ainsi renforcer les sites intégrés grâce à une bonne liaison avec les transports en commun, en dirigeant les investissements dans le petit commerce dans des proportions plus importantes que jusqu'à présent. De plus, la structure de Leipzig a été revue, les centres potentiels disponibles ont été développés. On a constitué différentes catégories de centres qui doivent former les nouveaux pôles d'attraction de la ville. Pour Leipzig, ce concept des centres prévoit trois niveaux :

- le centre-ville avec une zone d'attraction sur l'ensemble de la ville et de la région urbaine,

- des centres de quartier pour 30000 à 80000 habitants (7 au total),

- des centres de proximité pour 5000 à 25000 habitants (31 au total).

Ainsi, la structure du commerce de détail de Leipzig est fondée sur un système polycentrique hiérarchisé.

Le centre-ville joue un rôle prééminent en tant que centre de commerce pour toute la région de Leipzig-Halle-Dessau (fig. 3). Afin de le rendre plus attractif, même pour les grandes entreprises commerciales, on a réalisé à Leipzig le projet «Promenaden Hauptbahnhof » (un centre commercial intégré dans la gare du centre-ville). La gare principale de Leipzig se trouve sur la bordure nord du centre-ville (fig. 3 et photo 1) et constitue un pôle commercial extrêmement bien relié au réseau de transports en commun.

Figure 3 : Zones piétonnes et galeries-marchandes à Leipzig-centre

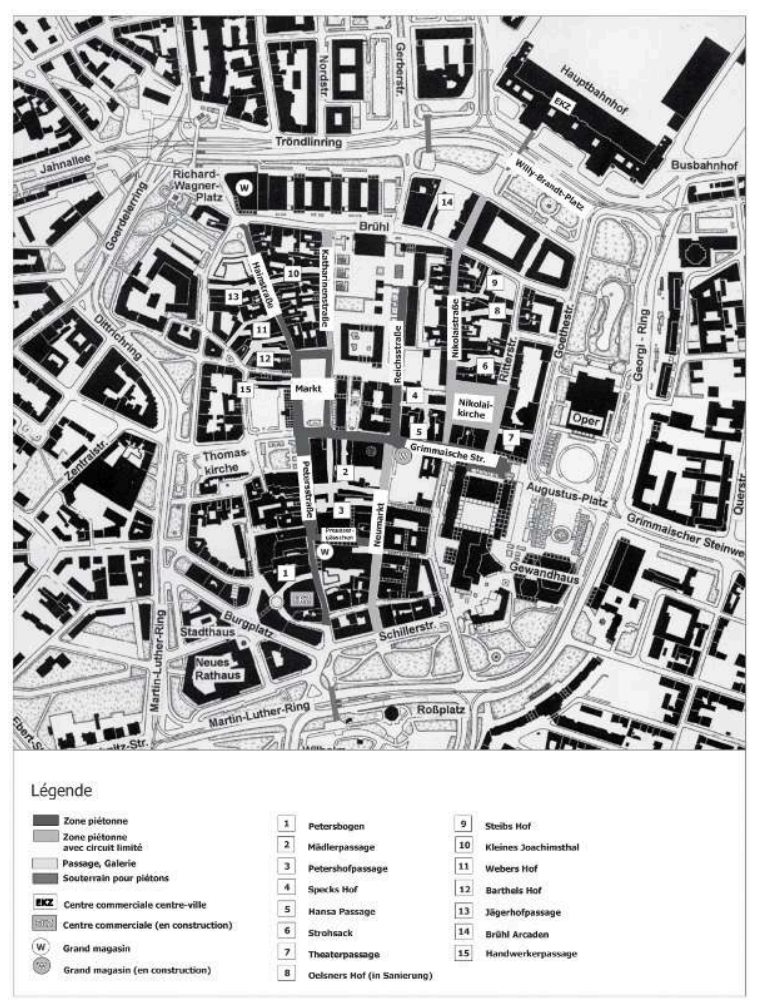


Photo 1 : Le centre commercial intra-urbain «Promenaden im Hauptbahnhof » sur la bordure nord du centre-ville

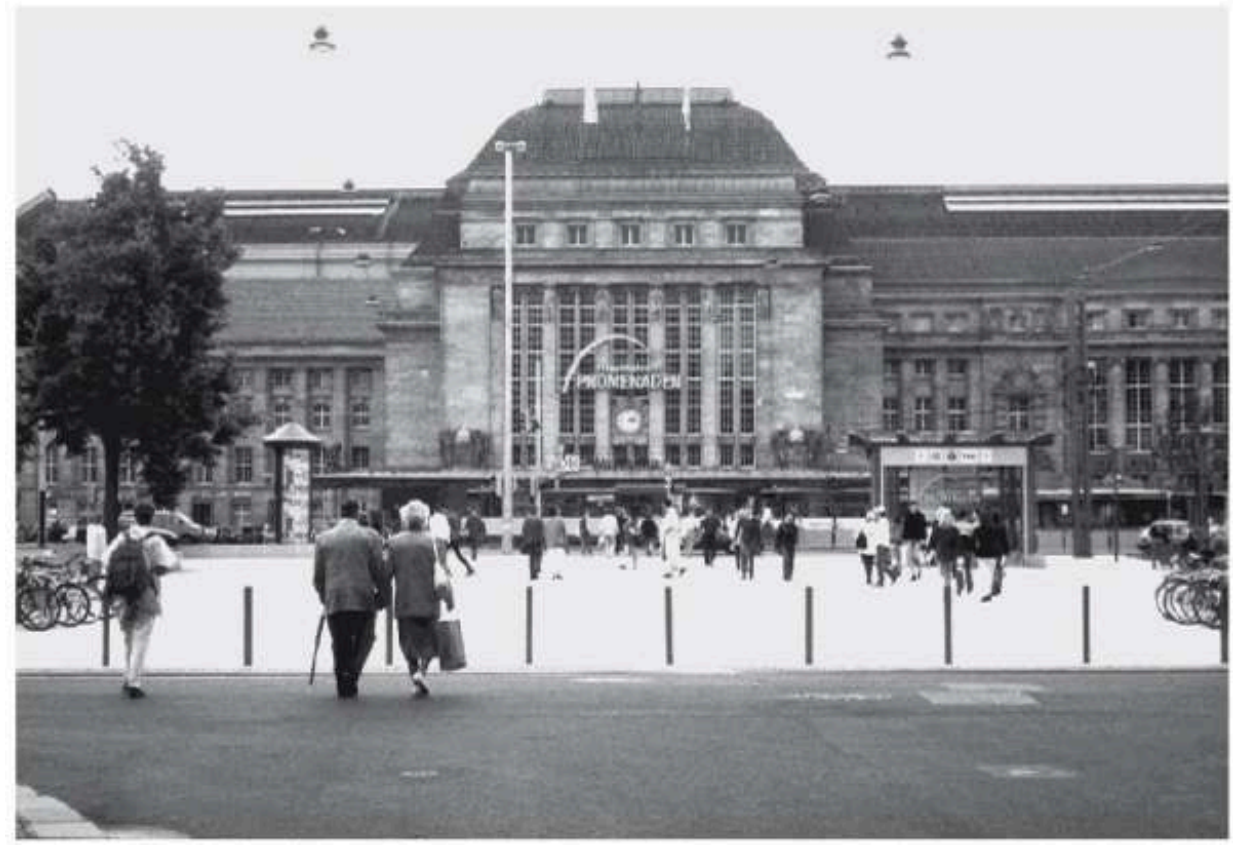

Photo : Hoppmann 1998

Les craintes que le développement de ce pôle se fasse au détriment du centre-ville ne se sont pas confirmées car, le plus souvent, les clients associent les achats dans le «Promenaden Center Hauptbahnhof» avec une visite du centre-ville. Le centre commercial de la gare est relié par un tunnel avec les rues commerçantes principales du centre-ville, si bien qu'on peut atteindre celles-ci en moins de cinq minutes de marche à pied. Ainsi, contre toute attente, cette réussite de la revalorisation du centreville comme centre commercial a rendu celui-ci de nouveau si attractif pour les entreprises commerciales déjà installées, que de nouveaux sites d'implantation de magasins ont pu être trouvés et que d'autres projets sont en cours de réalisation dans le centre (photo 2). 
Photo 2 : Construction de la nouvelle galerie Kaufhof (achevée en octobre 2001)

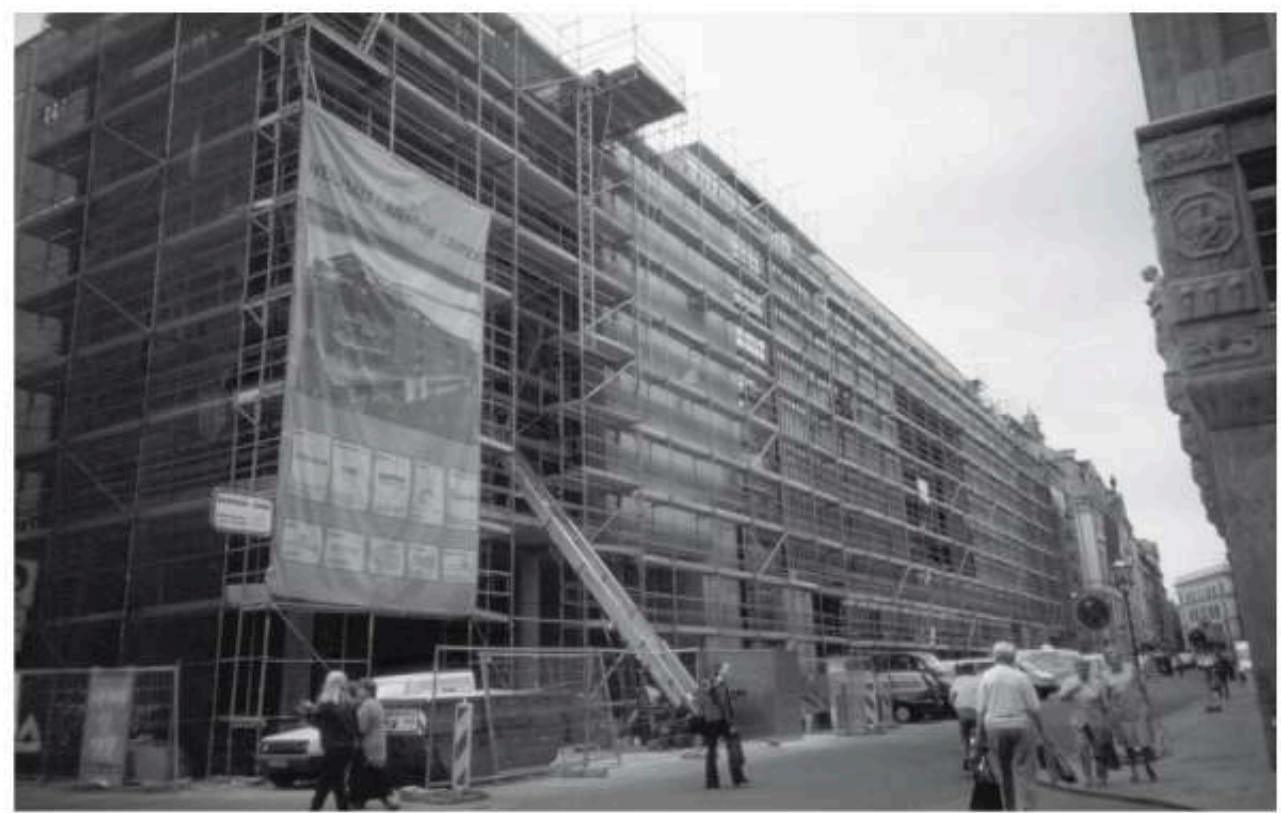

Photo : Hoppman 2001

21 Il ne faut pas s'imaginer les centres des villes allemandes sans les grands magasins; traditionnellement, ceux-ci ont toujours été installés dans les centres et forment un important pôle d'attraction pour les clients. À Chemnitz et à Dresde, les grands magasins constituent également une stratégie permettant de restituer au centre-ville son attractivité. À Chemnitz, les mesures de revitalisation doivent restituer un centre à la seule commune d'Europe considérée comme étant dépourvue de centre (Freie Presse du 6/12/99). Le concept développé, d'abord limité au centre-ville, prévoit la construction de galeries marchandes, de centres commerciaux intégrés et de magasins spécialisés. Comme les deux autres grandes villes de Saxe, Chemnitz a également eu à souffrir de l'anneau de centres commerciaux périphériques. Après la réunification, les investissements sont d'abord allés vers les sites périurbains, le centre-ville (même si, ici, il n'existe pratiquement pas) a là aussi été le perdant de cette course à l'investissement. Avant la Seconde Guerre mondiale, Chemnitz était un centre commercial traditionnel. Le grand magasin Tietz qui a ouvert en 1913 représente ainsi un pionnier de la nouvelle forme de commerce de détail en Allemagne. Puis, en 1929-1930 ce fut l'ouverture du grand magasin Schocker, précurseur des magasins de Stuttgart et de Nuremberg. Des trois magasins Schocker, seul celui de Chemnitz a survécu. Depuis la réunification, les deux grands magasins de Chemnitz ont été repris par la société Kaufhof et sont restés jusqu'à il y a peu de temps le seul point d'attraction du centre de Chemnitz. Avec le nouveau slogan « Mut zur Mitte» (Courage au centre) il est question de construire de nouveau un grand magasin à Chemnitz, le «magasin de verre », un projet développé également par la société Kaufhof. Une autre stratégie de la ville de Chemnitz est la mise en place d'un manager du centre qui doit réunir les intérêts des entreprises et des habitants du centre-ville.

En tant que centre d'activités commerciales, la ville de Dresde a également perdu en importance depuis la réunification en raison de l'installation massive des grandes surfaces en périphérie. D’après Pütz (1997), jusqu'en 1996, la surface de vente située dans le centre-ville s'est abaissée à 15 \% du total des surfaces commerciales. En 1996, la 
ville de Dresde avait une surface de vente totale de $615000 \mathrm{~m}^{2}$. Dans le même temps, les surfaces de vente en positions périphériques se sont élevées à presque $40 \%$. En 1992 (Stadt Dresden 199242 in Pütz 1997) le service de la planification urbaine considérait comme optimale la situation inverse (Pütz 1997). Depuis 1999, le déclin persistant du centre de Dresde comme centre d'activités commerciales a pu être stoppé, par la réalisation de deux grands magasins sur le vieux marché et par la revitalisation des magasins de la Königsstraße. Grâce à d'importants investissements publics et privés, des centres de services modernes sont actuellement en cours de réalisation. En dehors des activités de bureaux, des hôtels et des restaurants, ceux-ci rassemblent surtout de nombreux magasins avec une spécialisation dans le haut de gamme. Aussi, avec la reconstruction du marché de Dresde détruit pendant la seconde guerre mondiale, ainsi qu'avec la réalisation du centre commercial du complexe Frauenkirche, le centre-ville récupèrera des positions dominantes dans les groupes de branches importantes relevant du centre. La création de nouvelles zones piétonnes dans les trois grandes villes saxonnes est une stratégie de revitalisation qui doit être continuellement poursuivie dans les années à venir.

\section{Conclusion}

«Fidéliser les clients avec de nouveaux concepts car, quand on est content on achète finalement plus ", c'est le résultat d'une étude menée à Chemnitz sur la clientèle du commerce de détail. L'objectif de la politique de développement urbain dans les villes est-allemandes est de renforcer le rôle des centres supérieurs et de développer la compétitivité des centres intra-urbains. Par une stratégie de rénovation et de construction de centres commerciaux intégrés au tissu urbain qui leur est propre, les trois grandes villes saxonnes, Dresde, Chemnitz et Leipzig ont réussi à contrebalancer la concurrence des grands centres commerciaux périphériques non intégrés. Il faut souligner le fait que, dans les trois grandes villes, les concepts destinés à revitaliser le commerce de détail intra-urbain ont été développés en prenant en compte les acteurs de la ville, les entrepreneurs et les investisseurs, les demandes et les attentes des citoyens, les associations et les sociétés. On a renoué par exemple avec la vieille tradition des passages et des galeries. Par là même, on a accru les surfaces de vente dans les centres-villes et de cette façon, on a restitué aux villes leur spécificité et leur caractère unique par rapport aux infrastructures situées " auf der grünen Wiese ». Il se développe de nouveau des espaces urbains intéressants et divers, propices à la flânerie et qui satisfont les demandes des différents groupes d'utilisateurs (photos 3, 4, 5). Bien que le commerce de détail soit le facteur décisif de constitution des centres, il est couplé avec d'autres fonctions relevant du centre, comme les infrastructures culturelles, de formation et de loisirs. C'est ainsi que peut être accrue l'attractivité des centres-villes lors des achats. Le développement du centre-ville constitue la première priorité. Comme auparavant, il constitue l'espace d'identification pour les habitants de la ville et de la région. Il est le but et l'espace vécu pour les visiteurs et les touristes. Les obstacles typiques aux mutations dans le commerce de détail diminuent peu à peu d'importance. La nouvelle orientation du commerce de détail détermine une réévaluation significative des centres-villes. Un accord préalable des acteurs concernés, propriétaires immobiliers, investisseurs et urbanistes est pour cela incontournable. 
Photo 3 : Le centre commercial intra-urbain « Petersbogen » au cœur du centre-ville

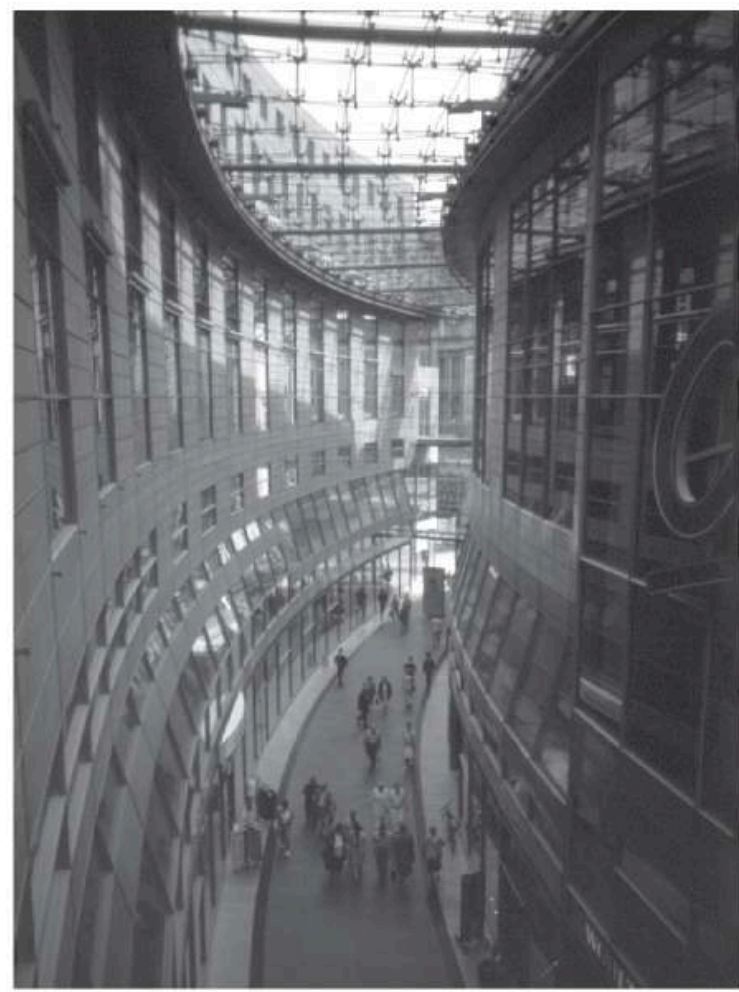

Photo : Maike Hoppmann 2001

Photo 4 : Zone piétonne de la Nikolaistraße

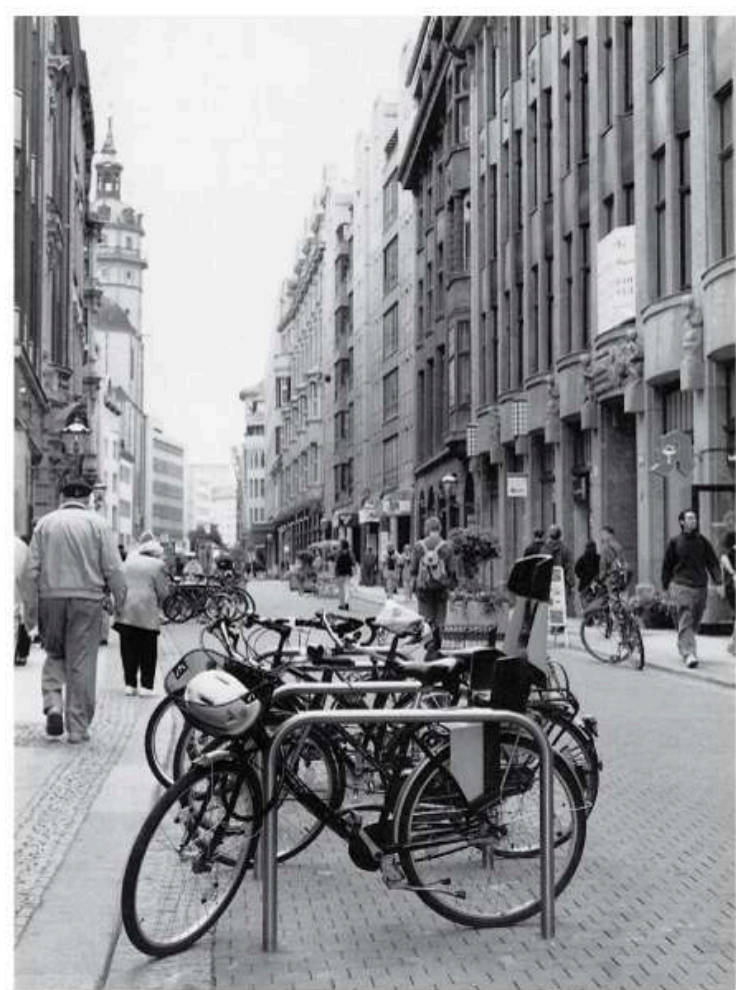

Photo : Stadtplanungsamt 1998 
Photo 5 : Terrasse de café au Nikolaikirchhof avec vue sur le passage Speck's Hof

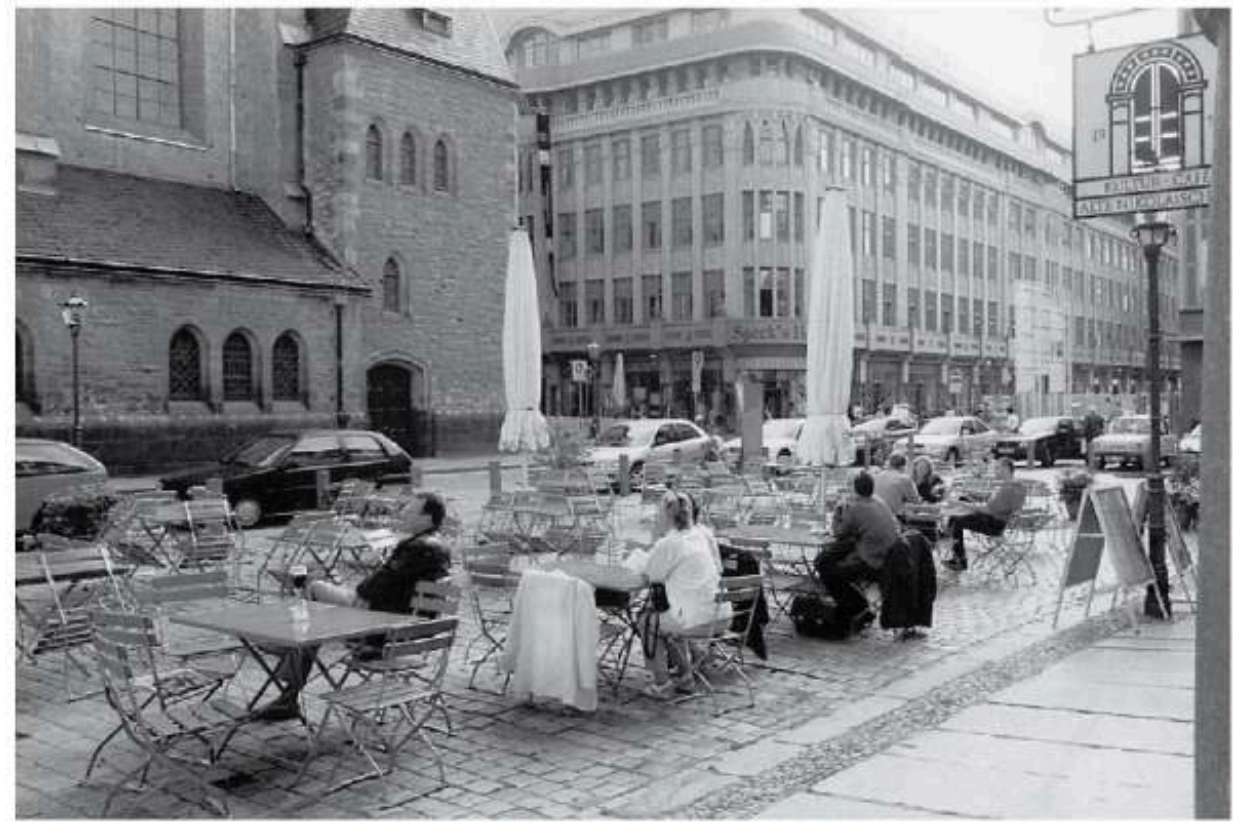

Photo : Stadtplanungsamt, 1998

\section{BIBLIOGRAPHIE}

Euro Handels Institut (Hrsg) (1998). - Shopping-Center-Planungsunterlagen. EHIFachdokumentation, Stand Juli 1998. Köln.

Gormsen Nils (1996). - Leipzig - Stadt, Handel, Messe. Die städtebauliche Entwicklung der Stadt Leipzig als Handels- und Messestadt. Daten - Fakten - Literatur zur Geographie. Institut für Länderkunde. Leipzig.

Jürgens Ulrich (1994). - Postsozialistische Transformation der Einzelhandelsstrukturen in Leipzig. In : Erdkunde Band 48/1994. Ss 302-314.

Jürgens Ulrich (1995). - Großflächiger Einzelhandel in den neuen Bundesländern und seine Auswirkungen auf die Lebensfähigkeit der Innenstädte. In : Petermanns Geographische Mitteilungen 139, 1995/3, Ss. 131-142.

Jürgens Ulrich (1996). - Einzelhandel in den neuen Bundesländern unter besonderer Berücksichtigung der Entwicklung großflächiger Handelsbetriebe - dargestellt anhand der Beispiele Leipzig und Rostock. In : Berichte zur deutschen Länderkunde. Bd. 70, Heft 2, 1996. Ss 327-346.

Jürgens Ulrich (1998). - Einzelhandel in den neuen Bundesländern - die Konkurrenzsituation zwischen Innenstadt und « Grüner Wiese » dargestellt anhand der Entwicklungen in Leipzig, Rostock und Cottbus. Kieler Geographische Schriften. Bd. 98. Kiel. 
Kulke Elmar (1997). - Effects of the economic transformation process on the structure and locations of retailing in East Germany. In : Journal of Retailing and Consumer Services 4 (1). Ss 49-55.

Mayer Günter, Pütz Robert (1997). - Transformation der Einzelhanmdelsstandorte in ostdeutschen Großstädten. In : Geographische Rundschau, H 3211/9/1997, S. 492-498.

Pütz Robert (1997). - Der Wandel der Standortstruktur im Einzelhandel der neuen Bundesländer Das Beispiel Dresden. In : Meyer, Günter (Hrsg.) 1997 : Von der Plan- zur Marktwirtschaft.

Wirtschafts- und Sozialgeographische Entwicklungsprozessen in den neuen Bundesländern. Mainzer Kontaktstudium Geographie, Bd. 3. Mainz.

Pütz Robert (1999). - Einzelhandel im Transformationsprozess. Das Spannungsfeld von lokaler Regulierung und Internationalisierung am Beispiel Polen. Geographische Handelsforschung Bd. 1. Passau.

Schmidt Helga, Fliegnerl (1997). - Der Saalepark - Güntherdorf ein Megaprojekt des Dienstleistungssektors im suburbanen Raum Leipzig - Halle : Wirkungen und Probleme. In : Wolf, Klaus und E. Tharun (Hrsg.) 1997, Einzelhandelsentwicklung (Beiträge zum Symposium in Frankfurt am Main im November 1995). Materialien 21. Institut für Kulturgeographie, Stadt- und Regionalforschung der J. W. Goethe-Universität Frankfurt am Main. Frankfurt am Main

Stadt Dresden, Dezernat für Stadtentwicklung (Hrsg.) (1992). - Rahmenkonzept Stadtentwicklung, Dresden.

Stadt Leipzig (1998). - Bericht zur Stadtentwicklung Leipzig 1998. Beiträge zur Stadtentwicklung 20. Stadt Leipzig, Dezernat Planung und Bau. Ss 33-35.

Stadt Leipzig (1999). - Stadtentwicklungsplan Zentren. Entwurf April 1999. Unveröffentlicht.

Staatsministerium für Umwelt und Landesentwicklung Freistaat Sachsen (1994). Landesentwicklungsbericht 1994. Dresden.

\section{RÉSUMÉS}

Depuis la réunification allemande en 1990, le commerce de détail des nouveaux Länder a été complètement restructuré. Cette restructuration menée par des entreprises commerciales ouestallemandes a conduit à une concurrence entre les centres-villes et les implantations extraurbaines « auf der grünen Wiese ». Dans cette contribution, on traite de l'évolution du commerce de détail à Leipzig comme exemplaire de celui d'une grande ville saxonne. Tandis que les implantations périphériques ont pu enregistrer une croissance des surfaces de vente, les centresvilles des nouveaux Länder ont subi une énorme perte de fonction. Ce déséquilibre doit être combattu par la mise en place d'un concept de centres de quartiers dans le cadre du plan de développement urbain de Leipzig. L'objectif de ce concept est la création de centres de différentes natures qui doivent constituer de nouveaux pôles d'attraction pour l'ensemble de la ville. Dans l'avenir, le centre-ville doit jouer un rôle prééminent comme lieu d'achat pour la région de Halle-Leipzig-Dessau. Afin de le rendre plus attractif également pour les grandes entreprises on a réalisé à Leipzig un centre commercial intra-urbain, les «Promenaden Hauptbahnhof ». Grâce à cette revalorisation du centre-ville de Leipzig, celui-ci est redevenu de nouveau si attractif pour les grands magasins déjà installées que de nouvelles implantations ont pu être trouvées et que d'autres projets sont en cours de réalisation dans le centre-ville. A Dresde et à Chemnitz, les centres-villes doivent également connaître une augmentation de leur attractivité grâce à des centres commerciaux intra-urbains et par l'installation des grands 
magasins traditionnellement implantés dans les grandes villes saxonnes. On peut décrire les stratégies développées à Dresde, Chemnitz et Leipzig comme étant un retour aux traditions en relation avec de nouveaux concepts. Au commerce de détail est ainsi dévolu un rôle porteur mais non exclusif. Seule une combinaison entre la fonction commerciale et les fonctions centrales relevant de la culture, de la formation et des loisirs peut de nouveau faire des centres-villes des lieux d'achat et d'identification pour les habitants d'une ville.

German reunification brought a complete alteration in the retail trade structures of the New Länder. This restructuring, forced by West German trading enterprises, led to a competitive situation between city centres and out-off town retailing. The aim of this contribution is to describe the urban development of Leipzig. Out-of-town retailing increased their sales areas until 1996, while city centres and traditional city districts were characterised by deficits. To counteract this imbalance a so-called «Stadtentwicklungsplan Zentren » is currently being brought into action as part of the urban planning guidelines. The aim of this programme is the embedding of centres within the urban boundary. Regeneration of the city centre as a main location of shopping is of prime importance. In order to attract retail capital to the city centre of Leipzig a project was initiated to bring the out-of-town'format into the centre. One of the projects carried out by private investors is the implementation of a shopping mall in the central railway station of Leipzig which is still in use. This increase in attractiveness triggered department stores (a main and traditional attraction of German city centres) to locate their facilities in the city centre. As in Leipzig, the town centre development of Dresden and Chemnitz is based on inner city shopping malls and department stores. Recollection of traditions as well as new concepts for retail trade structures, to counteract the loss of city functions, are the new strategies of urban development in Leipzig, Dresden and Chemnitz. Since only a combination of these functions will transform city centres into places of urban life, the new strategies are not only concerned with retail trade but also with other functions of a city centre such as culture, education and leisure facilities.

Seit der politischen Wende 1990 wurde die Einzelhandelslandschaft in den neuen Bundesländern von Grund auf umstrukturiert. Diese Umstrukturierung, gefördert durch westdeutsche Handelsunternehmen, führte zu einer Konkurrenzsituation zwischen den Innenstädten und der Grünen Wiese. Beispielhaft wird in diesem Beitrag die Einzelhandelsentwicklung von Leipzig als einer sächsischen Großstadt aufgezeigt. Während periphere Standorte einen Verkaufsflächenzuwachs verzeichnen konnten, erlitten die Innenstädte und gewachsenen Stadtzentren der neuen Bundesländer bis 1996 einen enormen Funktionsverlust. Diesem Ungleichgewicht soll mit der Durchsetzung eines Stadtteilkonzeptes im Rahmen des Stadtentwicklungsplanes in Leipzig entgegengewirkt werden. Ziel dieses Konzeptes ist die Schaffung von Zentren unterschiedlicher Ausprägung, die neue räumliche Schwerpunkte in der Gesamtstadt bilden sollen. Zukünftig soll dabei die Innenstadt als Einkaufsstandort für die Region Halle-Leipzig-Dessau Vorrang haben. Um sie auch für große Unternehmen attraktiver zu machen, wurde in Leipzig das Projekt eines innerstädtischen Einkaufszentrum (Promenaden Hauptbahnhof) realisiert. Durch diese Aufwertung der Innenstadt wurde die Leipziger City für schon ansässige Warenhausunternehmen wieder so attraktiv, dass neue Standorte Für die Warenhäuser gefunden werden konnten und sich weitere Projekte in der Innenstadt in der Realisierung befinden. Auch in Dresden und Chemnitz sollen die Innenstädte eine Attraktivitätssteigerung durch innerstädtische Einkaufszentren und die Ansiedlung der in der sächsischen Großstädten traditionellen Warenhäuser erfahren. Man kann die Strategien in Dresden, Chemnitz und Leipzig mit einer Rückbesinnung auf alte Traditionen verbunden mit neuen Konzepten beschreiben. Dem Einzelhandel kommt dabei zwar eine tragende, aber nicht die alleinige Rolle zu. Nur eine Kombination der zentralrelevanten Funktionen Kultur, Bildung 
und Freizeit mit der Funktion Einkauf kann die Innenstädte wieder zu Orten des Einkaufs und der Identifikation der Bewohner einer Stadt machen.

\section{INDEX}

Schlüsselwörter : Aufwertung der Innenstadt, Einkaufsstandorte auf der Grünen Wiese, Einzelhandel, innerstädtische Einkaufszentren

Keywords : inner-city shopping malls, out-of-town retailing, regeneration of the city centre, retail trade

Mots-clés : centres commerciaux intra-urbains, commerce de détail, implantations commerciales extra-urbaines, revalorisation du centre-ville

\section{AUTEURS}

\section{MAIKE HOPPMANN}

Universität Leipzig - Institut für Geographie - Johannisallee 19a - 04103 Leipzig - Allemagne HELGA SCHMIDT

Universität Leipzig - Institut für Geographie - Johannisallee 19a - 04103 Leipzig - Allemagne 